\title{
Neural Systems for Solving the Inverse Problem of Recovering the Primary Signal Waveform in Potential Transformers
}

\author{
Nikola Kasabov ${ }^{1}$ Senior Member IEEE, Gancho Venkov ${ }^{2}$ and Stefan Minchev ${ }^{3}$ Student Member,IEEE \\ ' Knowledge Engineering and Discovery Research Institute \\ Auckland University of Technology \\ Private Bag 92006, Auckland 1020, New Zealand \\ Emails: nkasabov@aut.ac.nz; qsong@aut.ac.nz \\ ${ }^{2}$ Technical University of Sofia, Bulgaria
}

\begin{abstract}
The inverse problem of recovering the potential transformer primary signal waveform using secondary signal waveform and information about the secondary load is solved here via two inverse neural network models. The first model uses two recurrent neural networks trained in an off-line mode. The second model is designed with the use a Dynamic Evolving Neural-Fuzzy Interface System (DENFIS) and suited for on-line application and integration into existing protection algorithms as a parallel module. It has the ability of learning and adjusting its structure in an on-line mode to reflect changes in the environment. The model is suited for real time applications and improvement of protection relay operation. The two models perform better than any existing and published models so far and are useful not only for the reconstruction of the primary signal, but for predicting the signal waveform for some time steps ahead and thus for estimating the drifts in the incoming signals and events.
\end{abstract}

\section{INTRODUCTION}

The growing of power system complexity and size leads to an increased importance of protective relays. An accurate reproduction of the real signals from the power system is needed. Iron-core measurement transformers are not ideal, because of the non-linearity of their excitation characteristics and their ability to retain large flux levels in their cores, known as remanent flux. The distorted waveforms can introduce large uncertainties, leading to delays or malfunction in relay tripping It is important to investigate the deformation of the real signals, caused by the transformers, and to recover the primary signals using the secondary ones.

Electric power systems are subjected to many types of disturbances that result in electrical transients due to faults or routine switching operations. During the first few cycles following a power system fault, high-speed protective relays are expected to make a correct decision as to the presence and location of the fault in order to preserve system stability and to minimize the extent of equipment damage. The dynamic performance of the protective relays depends to the large extent on the signals produced by the potential transformers (PT) and the current transformer (CT). Power system faults or other transients can cause saturation in the PT and CT, leading to distortion of their signals. The PT and $\mathrm{CT}$ transient errors can have a major impact on the security of the protective relays and can cause relay protection misoperations; unacceptably delayed operations or failures to operate.

Additional algorithms for prediction of PT primary signal for a few time samples ahead can increase the operational speed and reliability of the existing protection algorithms that would result in an improved decision-making and an increased overall performance of the relay protection.

There are several methods used so far to achieve the above goal. The methods of neural networks (NN), fuzzy systems (FS) and knowledge engineering have been used extensively for solving prediction and control problems across areas of applications [1]. NN-based predictors have been developed for systems, governed by linear models [2]. A recurrent NN predictor of power transformer magnetizing current, using terminal voltages as input values is realized in [3]. In [4] a reconstruction of primary current waveform for partially saturated CT is suggested via logical block and 3layered time-delay feed-forward NN (FNN). The use of FNN for CT saturation correction is considered in [5]. The CT is modelled in inverse direction by the FNN. In [6] and [7] the reproduction of CT primary current is considered, assuming steady state conditions for the magnetic flux and without remanent flux.

Despite of the successful applications of the above systems, there is a need for on-line learning and adaptive prediction techniques. One paradigm introduced for this purpose is called evolving connectionist systems (ECOS) [8]. ECOS evolve their structure and functionality through on-line, incremental, life-long learning from incoming data, thus being able to better predict future events. For the purpose of improving the relay protection performance we are introducing here the use of Dynamic Neuro-Fuzzy Inference Systems DENFIS [9], which is a specific prediction technique from the class of ECOS [8]. DENFIS is used here not only for the prediction and the recovery of the power system real current and voltage signals, but also for the prediction of future events. For initial training of the predictors, classical PT model is built first. Then the DENFIS predictor is used for on-line, real time learning and prediction. 
This paper is organized as follows: Section II presents the mathematical modelling of PT and its classical description as a non-linear dynamic system with hysteresis. Section III refers to the design of inverse model via two recurrent neural networks in a series. Simulation results that prove its adequate behaviour are shown. Section IV introduces a novel method for the design of neural-fuzzy predictors with the use of DENFIS. Verification test and simulation results are applied.

\section{Il. Mathematical Description of Potential. Transformers}

In this section a classical non-linear PT model with hysteresis is built for the purpose of generating training sequences that are used for the training the neural systems. The model is realized in MATLAB. The PT equivalent circuit is shown on fig. 1 .

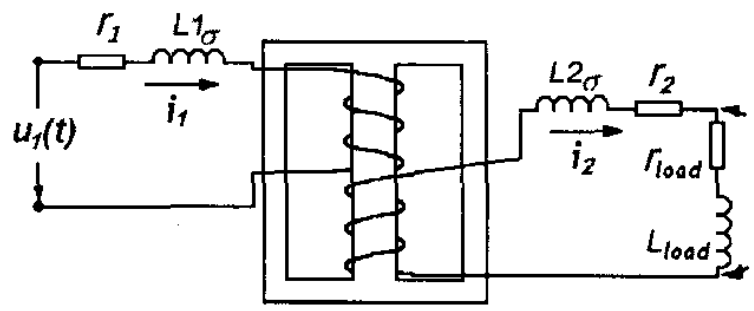

Fig. 1. Equivalent circuit of a single phase PT.

As a non-linear system with hysteresis, a 1-phase, 2winding PT can be described mathematically via non-linear system of differential-algebraic equations [10]:

$$
\begin{aligned}
& \dot{\varphi}_{1}(t)=-\frac{r_{1}\left(G_{j \mu}+L_{2 \sigma}+L_{\text {loud }}\right)}{D} \varphi_{1}(t)+\frac{r_{1} G_{p e}}{D} \varphi_{2}(t)+u_{1}(t) \\
& \dot{\varphi}_{2}(t)=\frac{\left(r_{2}+r_{\text {lowd }}\right) G_{\mu \omega}}{D} \varphi_{1}(t)-\frac{\left(r_{1}+r_{\text {load }}\right)\left(G_{\mu \omega}+L_{2 \sigma}+L_{\text {lood }}\right)}{D} \varphi_{2}(t
\end{aligned}
$$

where $\quad D=G_{\mu}\left(L_{\text {lo }}+L_{2 \sigma}+L_{\text {loud }}\right)+L_{1 \sigma}\left(L_{2 \sigma}+L_{\text {loud }}\right)$

and

$$
G_{\mu e}=\frac{s_{e}}{l_{e}} \mu_{D \gamma N}(B)=\frac{s_{e}}{l_{e}} f\left(\frac{G_{\mu e}\left(\left(L_{2 \sigma}+L_{2 o a d}\right) \varphi_{1}+L_{1 \sigma} \varphi_{2}\right)}{D}\right.
$$

The supplying voltage $u_{1}(t)$ is the excitation signal; $u_{2}(t)$ is the secondary voltage; $\Psi_{1}$ and $\Psi_{2}$ are flux linkages; $r_{1}$, $r_{2}, L_{1 \sigma}, L_{2 \sigma}$ are parameters of the windings; $B$ is magnetic flux density; $G_{\mu e}$ is the equivalent permeance of the magnetic core; $s_{e}$ and $l_{e}$ are geometrical parameters of the core; $r_{\text {load }}$ and $L_{\text {load }}$ are load resistance and inductance. The auxiliary variables $\varphi_{1}(t)=L_{1} \sigma_{1}(t)+\Psi_{1}(t)$ and $\varphi_{2}(t)=\left(L_{2 \sigma}+L_{\text {load }}\right) i_{2}(t)+\Psi_{2}(t)$ are introduced. The secondary voltage is calculated from:

$$
\begin{aligned}
u_{2}(t)= & r_{\text {load }}\left(-\frac{G_{\mu e}}{D} \varphi_{1}(t)+\frac{\left(G_{\mu e}+L_{l \sigma}\right)}{D} \varphi_{2}(t)\right)+ \\
& +L_{\text {load }}\left(-\frac{G_{\mu e}}{D} \frac{d \varphi_{1}(t)}{d t}+\frac{\left(G_{\mu e}+L_{1 \sigma}\right)}{D} \frac{d \varphi_{2}(t)}{d t}\right)
\end{aligned}
$$

The non-linear implicit relation (2) is given as data set $\mu_{D Y N}=f(B)$ and shown on fig. 2. According to [11] eddy current and hysteresis losses are combined into one core loss term, which is accounted for by a voltage-dependent resistance additional load $r_{2 h}=r_{2 h}\left(u_{2}(t)\right)$ on the PT secondary. This explicit relation is shown on fig. 3 .

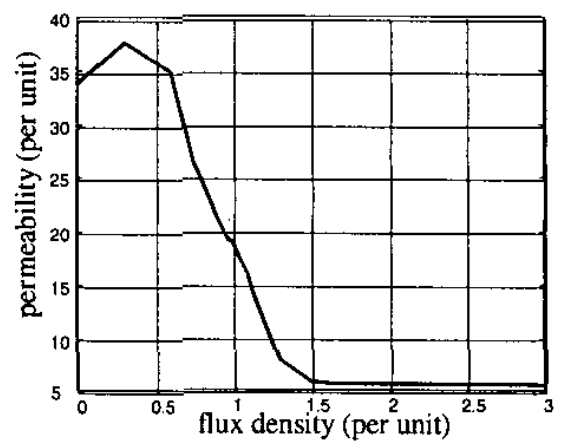

Fig. 2. The relation $\mu_{D Y N}=f(B)$.

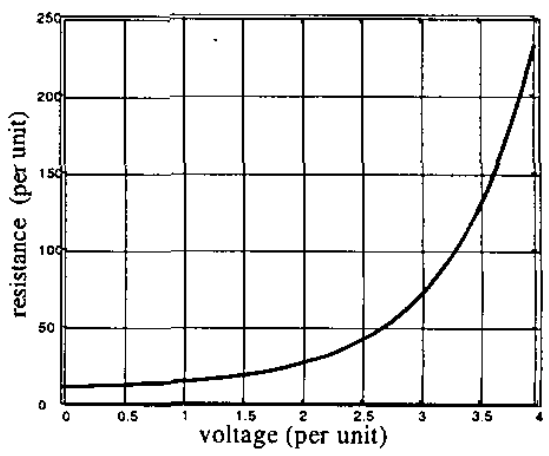

Fig. 3. The relation $r_{2 h}=r_{2 h}\left(u_{2}(t)\right)$. 
The classical PT model based on (1), (2), (3) and also based on the non-linear relations $\mu_{D Y N}=f(B)$ and $r_{2 h}=r_{2 h}\left(u_{2}(t)\right)$ is constructed for generation of training samples. Certain PT parameter values are chosen as follows: $U_{1}=220 \mathrm{~V} ; U_{2}=2200 \mathrm{~V} ; I_{1}=2 \mathrm{~A} ; I_{2}=1 \mathrm{~A}$ and the model parameter values are: $\quad r_{1}=2.991 \Omega$; $r_{2}=121.99 \Omega ; L_{1 \sigma}=0.051 \mathrm{mH} ; L_{2 \sigma}=2.45 \mathrm{mH}$; $r_{\text {load }}=20 \mathrm{k} \Omega ; L_{\text {load }}=1.27 \mathrm{H}$.

III. Inverse Model for Recovering Primary Signal Waveform BasED ON RECURRENT NEURAL NeTworks

The idea for construction of recurrent neural network (RNN) using FNN and feedbacks from its outputs to the inputs is presented in [12]. For the training phase the input to a FNN includes the excitation signal and the past values of the classical model output. We assume that after a suitable training the FNN gives a good representation of the original dynamic system. For the subsequent post-training purposes the FNN output itself (and its delayed values) can be fed back and used as a part of the NN input. In this way the initial FNN can be upgraded to time-delayed RNN and can be used independently of the original system.

The algorithm for construction of PT neural based inverse model has two steps. In the first step simulations with various excitations - supplying voltages $u_{1}(t)$ and different load parameters $\left(r_{\text {load }}, L_{\text {load }}\right)$ have been conducted and the corresponding classical model responses $\Phi(t)$ and $u_{2}(t)$ have been recorded. The excitations $u_{1}(t)$ are constructed for thorough characterization of the considered dynamic system. They are composed of sine waves (with fundamental frequency $50 \mathrm{~Hz}$ and different magnitudes from 1 to $1000 \mathrm{~V}$ ) and random noise with normal distribution. The sample time is $3.125 \times 10^{-4} s$ or 64 points pre fundamental frequency cycle $(0.02 \mathrm{~s})$. Furthermore, to cover more of the real secondary loads the simulations were performed with varying $r_{\text {load }}$ and $L_{\text {load }}$ and a sample time of $0.1 \mathrm{~s}$. The limits for the load resistance and inductance are $r_{2 \text { load }} \in[0.5 ; 5000] \Omega$ and $L_{\text {load }} \in[0.01 ; 10] H$. The excitations, the load variations and the corresponding classical model responses are used for construction of training sequences.

In the second step the two RNNs - NN1 and NN2 are trained independently, using standard backpropagation training technique, available in MATLAB. NN1 is for approximation of magnetic flux $\Phi(t)$ waveform. It has two hidden layers with neurons (10-10) having tansig activation functions. The output layer has one linear neuron. After the training error is driven to sufficiently small value (less than $1.0 \times 10^{-8}$ ) the training process is stopped. The parameters of the FNN are fixed and after inclusion of the feedback connections and buffers for 3 past values the training is finished. The second RNN- NN2 is for approximation of the primary voltage $u_{1}(t)$ waveform. It has two non-linear layers (17-12) with tansig activation functions and one linear output neuron. NN2 is constructed and trained in the same way like NN1. Then the two RNN are combined to work in series (fig.4) and they form a complex neural system for modeling of the PT inverse dynamics.

The designed neural system was tested thoroughly with wide range of signals and loads, different from those, used in the training samples. The neural inverse model approximates the classical model results with acceptable accuracy (less than $5 \%$ ). We found that the neural system has not only learned the training samples, but also exhibited a good capability for generalization beyond the training data.

To show the capabilities of the presented inverse model we have used an extreme case (not included in the training samples), the transformer load changes rapidly while the dynamics in the secondary voltage is considerable. This is a demonstration of the high non-linear inverse dynamics of the physical object, as it is driven to saturation levels.

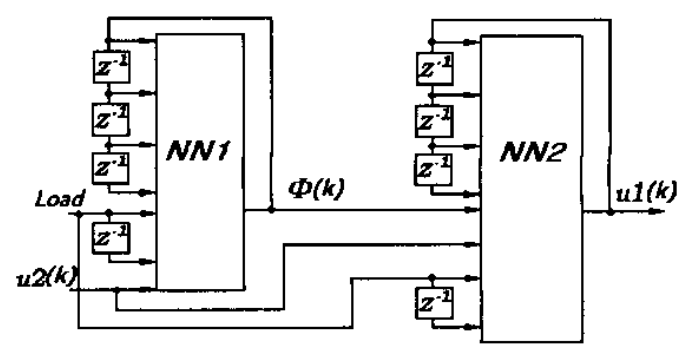

Fig. 4. Potential transformer inverse model architecture.

The PT inverse model, realized by means of RNN structures is described by:

$$
\begin{aligned}
& \Phi(k)=\gamma\left(\begin{array}{l}
\Phi(k-1), \Phi(k-2), \Phi(k-3), u_{1}(k), \ldots \\
\ldots r_{\text {load }}(k), r_{\text {load }}(k-1), L_{\text {load }}(k), L_{\text {load }}(k-1)
\end{array}\right) \\
& u_{2}(k)=\delta\left(\begin{array}{l}
u_{1}(k), \Phi(k), u_{2}(k-1), u_{2}(k-2), u_{2}(k-3), \ldots \\
\ldots r_{\text {load }}(k), r_{\text {load }}(k-1), L_{\text {load }}(k), L_{\text {load }}(k-1)
\end{array}\right)
\end{aligned}
$$

where $\gamma(\bullet)$ and $\delta(\bullet)$ are non-linear functions. 


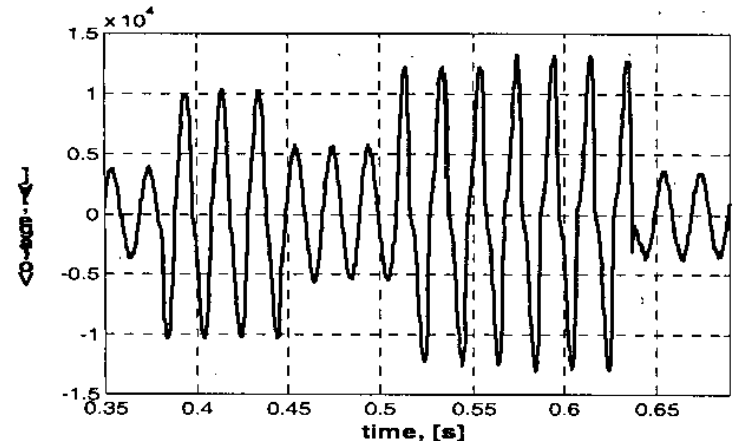

Fig. 5. Secondary voltage $u_{2}(t)$ the input signal to the neural model.
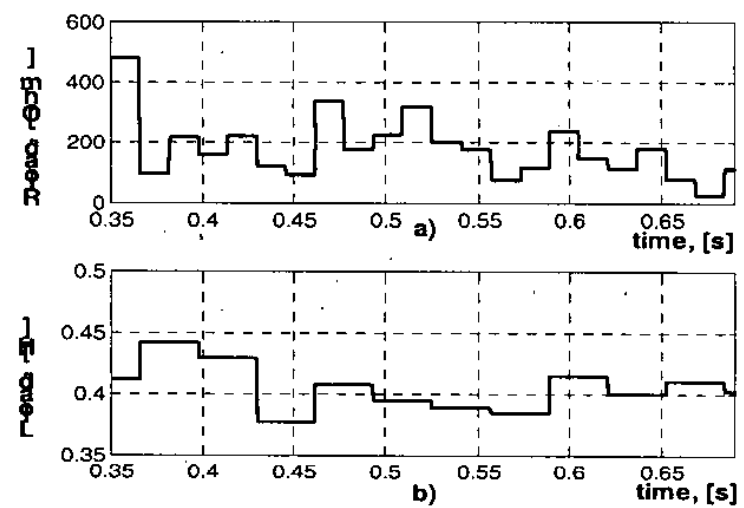

Fig. 6. Secondary load variations during the simulation test: a) load resistance $r_{\text {lood }}$; b) load inductance $L_{\text {load }}$.

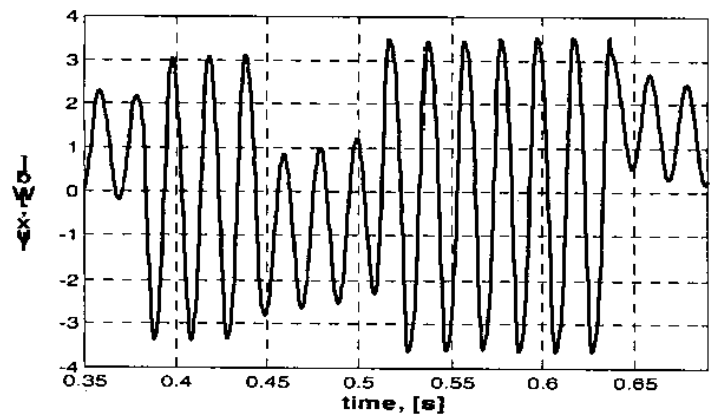

Fig. 7. Magnetic flux $\Phi(t)$ obtained from the neural model.

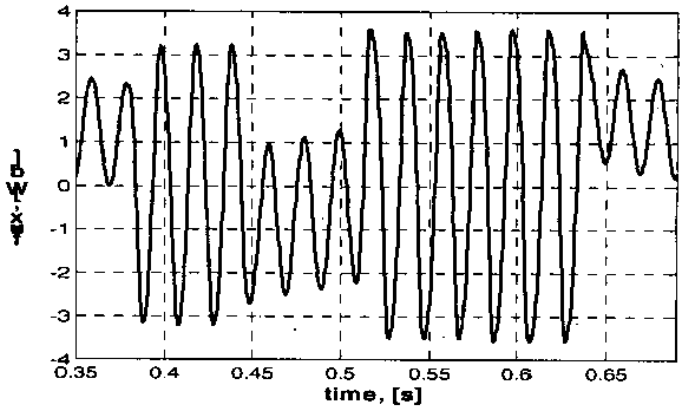

Fig. 8. Magnetic flux $\Phi(t)$ obtained from the neural model.

A simulation test results are presented on fig.5 - fig.12. The input signal $u_{2}(t)$ is shown on fig.5 and the load variations are on fig. 6 . The result from NN1 (fig.7) is approximation of the magnetic flux waveform and is in good agreement with the classical model dynamic trajectory, shown on fig.8. The reconstructed primary voltage waveform $u_{1}(t)$, obtained from the inverse neural model is on fig. 9 and the corresponding signal concerned with the classical model is on fig. 10 . The errors of the neural system versus the classical model reactions are depicted on fig. 11 .

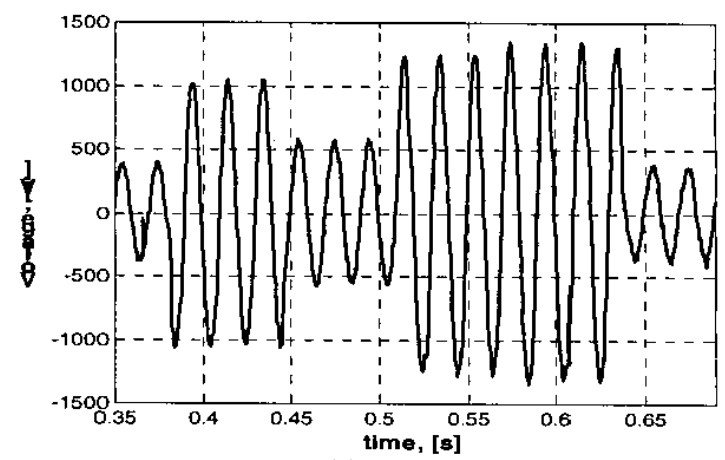

Fig. 9. Primary voltage $u_{1}(t)$ obtained from the neural model.

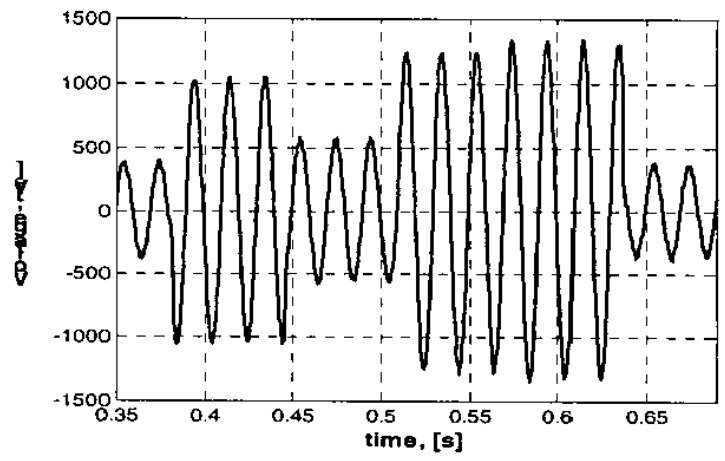

Fig. 10. Primary voltage $u_{1}(t)$ obtained from the classical model. 

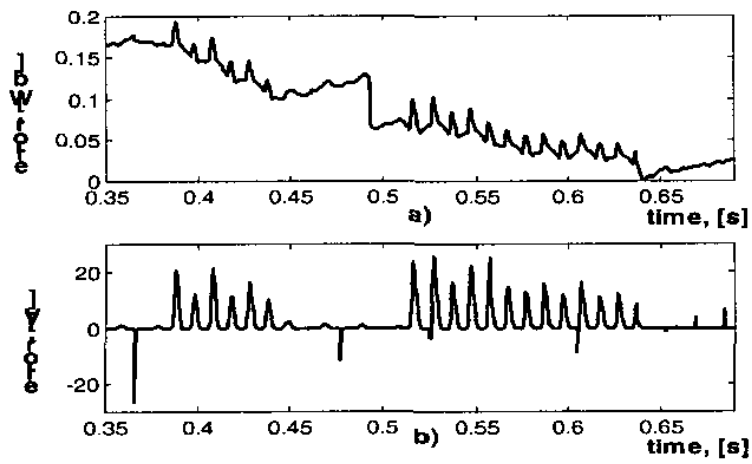

Fig. 11. The errors: a) in approximation of the magnetic flux; b) in approximation of the primary voltage.

IV. Primary Voltage Predictor Via Denfis Modelling

\section{A. DENFIS: Dynamic Evolving Neural-Fuzzy Interface System}

DENFIS belongs to the paradigm of the evolving connectionist systems (ECOS) [8]. DENFIS evolve through incremental learning and accommodate new input data through local element tuning [9]. New fuzzy rules are created and updated during the operation of the system. A new evolving clustering method is employed in DENFIS. At each time step, depending on the position of the input vector in the input space, the DENFIS output is calculated through a fuzzy interface system, based on $m$-most activated fuzzy rules, which are dynamically chosen from a fuzzy rule set. Papers and software written in MATLAB of several ECOS models that include DENFIS, can be found in [8] and also downloaded from www.kedri.info (the book ref. [8] entry) or from (www.spinger.de $\rightarrow$ the book ref. [8] entry).

\section{B. Problem Definition}

The diagram for prediction of PT input signal is shown on Fig. 12. The input value $x(k)$ is the primary signal at instant $k$. After sampling the secondary signal $y(k)$ together with information about the load are fed to the predictor. The aim is to predict the primary signal value $x(k+1)$ for some time sample ahead in the future and thus to predict possible drifts in the real power system voltages.

The construction algorithm has two steps. In the first step simulation with various signals - supplying voltages and different load conditions have been performed with the classical PT model. The input signal waveforms, the load variations and the corresponding secondary signal waveforms are recorded and used for construction of training sequences. The chosen sample time is $1.0 \times 10^{-3} \mathrm{~s}$ In the second step the DENFIS model is trained as an initial training and initial adjustment of its structure, as it will further adapt to new data in an on-line mode.

\section{Simulation Results}

The constructed DENFIS based predictor for primary voltage was tested with wide range of excitation signals and load conditions to prove its validity. To demonstrate its adequate operation, simulation results are shown below. Fig. 13 shows the desired and the predicted over time values of the PT primary voltage with the use of a DENFIS operating in an on-line learning mode. The prediction error is much lower that the error produced by other prediction models so far.

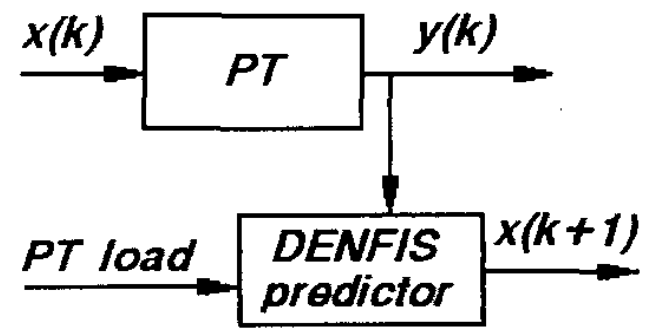

Fig. 12. Block diagrams for construction of PT primary signal predictor.

The designed predictor for estimation of $\mathrm{PT}$ primary voltage $u_{1}(t)$ waveform or power system real voltage, constructed according the diagram from Fig. 12 is suited for on-line operation. The testing results (fig. 13) show good coincidence of the two time series data with acceptable differences. The time series values are scaled with the primary voltage rated value

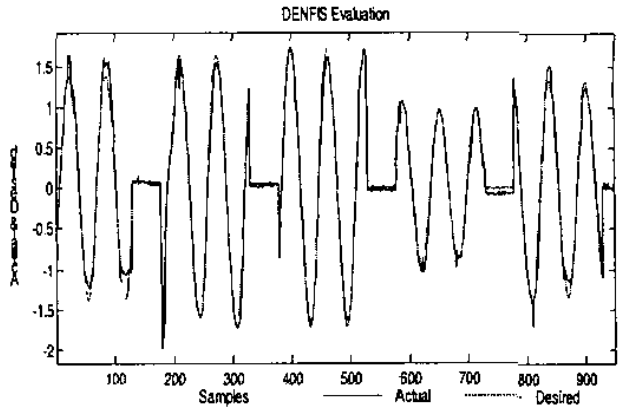

$(220 \mathrm{~V})$

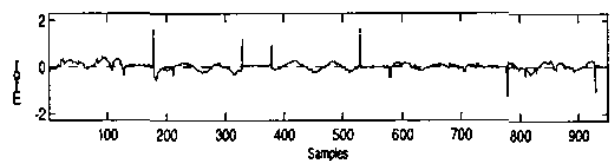

Fig. 13. PT primary voltage predictor results from verification test. 


\section{ConClusion}

It is well known (see [6], [7]) that an accurate estimation of the primary signal waveform using the measured secondary signal waveform can not be achieved in real time using conventional modeling methods, because of the complexity in modeling of transformers magnetic systems. The first NN inverse model based on two recurrent NN is trained in an off-line learning mode and has a great computational power and is suited for real-time application. The results show that one high non-linear dynamic system with hysteresis can be modelled successfully with NN system. The inverse PT neural model is adopted for being integrated into the existing digital protection ralays algorithms as a parallel function. The incorporation of the proposed routine in digital protective relaying algorithms provides much more accurate estimate of the real primary signal waveform than the usually achieved with using the PTs nominal ratio. The added element of artificial intelligence improves the protection relays reliability and increases the operational speed. The first model, based on RNN is difficult to train on new data in an on-line mode as it operates in a working environment.

The complexity and dynamics of power system transients require sophisticated methods and tools for building on-line adaptive intelligent systems for an improved relay protection operation. They should be able to adapt as they operate, and to refine their algorithms with possible environmental changes.

The second model in this paper is an application of a new fuzzy interface system, DENFIS for the design of an on-line primary voltage value predictor for relay protection purposes. It is suited for real time applications.

The two models perform better than any existing and published models so far and are useful not only for the reconstruction of the primary signal, but for predicting the signal waveform for some time steps ahead and thus for estimating the drifts in the incoming signals and events.

The analysis of the results indicates clearly the advantages of DENFIS when used for on-line applications for predicting input-output signals of high non-linear dynamic systems with hysteresis.

The ECOS [8] are not only adaptive learning systems, but also knowledge-based systems as they allow for rules to be extracted from a trained system. These rules can be further analyzed for a better understanding of the relation between environmental changes and power security protection.

\section{ACKNOWLEDGEMENT}

The work is supported through funding from the Technical University in Sofia, Bulgaria and from the Foundation for Research, Science and Technology of New Zealand (NERF grant AUT02/001). We would like to acknowledge Dr. Qun Song, Mrs Joyce D'Mello and Peter Hwang from the Knowledge Engineering and Discovery Research Institute (KEDRI) for their assistance.

\section{REFERENCES}

[1] N. Kasabov, Foundations of neural networks, fuzzy systems and knowledge engineering, The MIT Press, 1996.

[2] C. Alippi and V. Piuri, "Experimental neural networks for prediction and identification," IEEE Trans. on Instrumentation and Measurement, vol. 45 , no. 2 , pp. 670-676, 1996.

[3] I. Kamwa, et al, "Recurrent neural networks for phasor detection and adaptive identification in power system control and protection," IEEE Trans. on Instrumentation and Measurement, vol. 45, no. 2; pp. 657-663, 1996.

[4] J. Pihler, B. Grcar, D. Dolinar, K. Elissa, "Improved operation of power transformer protection using artificial neural networks," IEEE Trans. on Power Delivery, vol. 12, no. 3, pp. 1128-1136, 1997.

[5] D. C. Yu, J. C. Cummins, Z. Wang, H-J. Yoon, L. A. Kojovic, "Correction of current transformer distorted secondary currents due to saturation using artificial neural networks," IEEE Trans. on Power Delivery, vol. 16, no. 1, pp. 189-194, 2001

[6] N. Locci and C. Muscas, "Hysteresis and eddy currents compensation in current transformers," IEEE Trans. on Power Delivery, vol. 16, no. 2, pp. 154-159, 2001

[7] Y. C. Kang et al, "An algorithm for compensating secondary current of current transformers," IEEE Trans. on Power Delivery, vol. 12, no. 1 , pp. 116-122, 1997.

[8] N. Kasabov, Evolving connectionist systems: Methods and Applications in Bioinformatics, Brain Study and Intelligent Machines, Springer Verlag, London-New York, 2002.

[9] N. Kasabov, Q. Song, "DENFIS: Dynamic evolving neural-fuzzy interface system and its application for time-series prediction," IEEE Trans. on Fuzzy Systems, vol. 10, no. 1, pp. 144-154, 2002.

[10] S. Minchev, "A potential transformer digital model and parameters calculation via optimization procedures," Proc. of the XVII Int.Conf. Applications of Mathematics in Engineering and Economy, Sozopol, Bulgaria, pp. 485-496, 2001.

[11] X. S. Chen, P. Neudorfer, "Digital model of three phase five-legged transformer," IEE Proceedings $C$, vol. 139, no. 4, pp. 351-358, 1992.

[12] K. Hunt, D. Sbarbaro, R. Zbikowski and P. Gawthrop, "Neural networks for control systems - a survey," Automatica, vol. 28, no. 6, pp. $1083-1112,1992$. 